\title{
PNUTS knockdown potentiates the apoptotic effect of Roscovitine in breast and colon cancer cells
}

\author{
GABRIEL DE LEON, MARGARET CAVINO, MIKILYN D'ANGELO and NANCY A. KRUCHER
}

\author{
Department of Biology and Health Science, Pace University, 861 Bedford Rd, Pleasantville, NY 10570, USA
}

Received November 20, 2009; Accepted December 31, 2009

DOI: 10.3892/ijo_00000611

\begin{abstract}
The phosphorylation state of Retinoblastoma protein $(\mathrm{Rb})$ plays a role in cell proliferation and apoptosis. Within cells, cyclin dependent kinases (cdks) phosphorylate $\mathrm{Rb}$ in response to growth stimulatory signals, whereas protein phosphatase 1 (PP1) dephosphorylates Rb when cells stop proliferating or undergo apoptosis in response to antiproliferative or stress signals. Stimulation of PP1 activity via siRNA mediated knockdown of its interacting protein PNUTS (Phosphatase Nuclear Targeting Subunit) leads to Rb dephosphorylation and apoptosis in cancer cells. We utilized two separate methods to modulate the phosphorylation state of $\mathrm{Rb}$ in cancer cells. Kinase activity toward $\mathrm{Rb}$ is inhibited by the clinically relevant cdk inhibitor, Roscovitine. In addition, siRNA mediated PNUTS knockdown stimulates phosphatase activity toward $\mathrm{Rb}$. Either of these treatments in cancer cells causes a 2-fold stimulation of apoptosis. When activation of phosphatase activity is combined with inhibition of cdk activity toward $\mathrm{Rb}$, however, cells exhibit a 4-fold increase in apoptosis. The mechanism by which PNUTS knockdown mediated PP1 activation leads to apoptosis was determined to be dependent on the activity of the transcription factor E2F1. The $\mathrm{Rb}$ phosphorylation profiles resulting from each treatment were analyzed and found to be similar but not identical. In addition, the two treatments differentially effect the expression of bcl-2 family proteins. Thus inhibition of cdk activity and activation of PP1 activity toward pRb are functionally distinct processes that together increase the apoptotic effect in cells.
\end{abstract}

\section{Introduction}

Apoptosis is a highly controlled process utilized by multicellular organisms during development or later within the adult organism to clear damaged cells in a manner that minimizes disruption to nearby cells (1). Many cancer cells acquire the ability to evade apoptosis which results in the persistence of

Correspondence to: Dr Nancy A. Krucher, Department of Biology and Health Science, Pace University, 861 Bedford Rd, Pleasantville, NY 10570, USA

E-mail: nkrucher@pace.edu

Key words: Rb, cdk, Roscovitine, PNUTS, E2F1 damaged cells which can contribute to tumorigenesis (2). The $\mathrm{Rb}$ tumor suppressor protein $(\mathrm{Rb})$, in addition to its functions in cell proliferation, differentiation and senescence, is also an important regulator of apoptosis (3). Evidence for this notion was initially revealed using $\mathrm{Rb}$-null mice which exhibit excessive apoptosis in the nervous system, lens, and skeletal muscles (4-6). Subsequently it was shown that expression of exogenous $\mathrm{Rb}$ in SAOS-2 Rb-null cells blocked apoptosis in response to radiation exposure (7). Finally it was demonstrated that apoptosis triggered by DNA damaging agents could be blocked in an Rb-dependent manner (8). These data have led to the notion that $\mathrm{Rb}$ plays an important role in apoptosis.

The function of $\mathrm{Rb}$ in the control of cell division is regulated by phosphorylation on several amino acids (9). In proliferating cells, $\mathrm{Rb}$ phosphorylation status is controlled by the cyclin dependent kinases (cdks) which phosphorylate $\mathrm{Rb}$ in response to growth stimulatory signals and protein phosphatase 1 (PP1) which dephosphorylates $\mathrm{Rb}$ at the end of each $\mathrm{M}$ phase $(9,10)$. In the absence of proliferative stimuli, cdk activity is inhibited, and $\mathrm{Rb}$ is unphosphorylated which leads to cell cycle arrest. The anti-proliferative activity of $\mathrm{Rb}$ is mediated via interaction with the E2F family of transcription factors which regulate both the G1 to $S$ phase transition and stimulation of apoptosis $(9,11)$. However, when proliferating cells are treated with apoptotic stimuli, $\mathrm{Rb}$ becomes dephosphorylated due to the activation of PP1 which can lead to cell cycle arrest and/or apoptosis (12-14). Activity of PP1 is controlled by association with regulatory subunits responsible for the substrate specificity, localization and activity of the PP1 catalytic subunit (15). PNUTS (Phosphatase Nuclear Targeting Subunit) is a PP1 binding protein that regulates PP1 activity toward Rb (16-18). When PNUTS expression is reduced by siRNA in colon, breast and ovarian cancer cells, apoptosis is induced due to activation of PP1 and dephosphorylation of Rb (19). The effect of PNUTS knockdown is dependent on the expression of $\mathrm{Rb}$. In addition, PNUTS is involved in controlling cell death in response to cell stress such as hypoxia (20).

In this study, in order to examine the effect of targeting both cdk and PP1 activity toward Rb in cancer cells, we combined PNUTS siRNA with cdk inhibition using the clinically relevant cdk inhibitor Roscovitine (21). Roscovitine (CYC 202, Seliciclib) is a purine analog that competes with ATP for binding to the active site of cdks and exhibits potent in vitro activity against $\mathrm{cdk} 1, \mathrm{cdk} 2, \mathrm{cdk} 5, \operatorname{cdk} 7$ and $\mathrm{cdk} 9$ (22-24). Downstream targets of cdks include proteins involved 
in the control of cell proliferation and cellular transcription. For example, Roscovitine-mediated cdk inhibition prevents phosphorylation of $\mathrm{Rb}$ and induces cell cycle arrest in several model systems (25-27). Depending on dose and cell type, Roscovitine can also induce apoptosis $(28,29)$. In addition, Roscovitine inhibits phosphorylation of the carboxy-terminal domain of the large subunit of RNA polymerase II, which is required for transcriptional activity (28). Some studies have found that the mechanism of action of Roscovitine involved the activity of the tumor suppressor gene p53 (30-32). Finally, Roscovitine has been shown to induce cell cycle arrest or apoptosis in combination with other treatments such as doxorubicin, ErbB targeting agents, TRAIL induced apoptosis and inhibition of farnesyl protein transferase (33-36). Thus it appears that Roscovitine affects multiple targets within cell cycle and transcription activation pathways and that the best clinical utilization of Roscovitine may be in combination with other agents.

In this study we show that either cdk inhibition or PP1 activation alone can induce apoptosis in cancer cells. However, when utilized in combination, the effect on cell number and apoptosis in breast and colon cancer cells is enhanced by approximately 2 -fold. The mechanisms involved in each of these processes is similar in that both appear to require the activity of E2F1, however, each process targets a different subset of $\mathrm{Rb}$ phosphorylation sites and each leads to a different pattern of expression of the bcl-2 protein family members which carry out apoptosis. Finally, the ability of PNUTS knockdown to potentiate the effect of Roscovitine appears to occur independent of p53 status in the cells utilized.

\section{Materials and methods}

Cell culture. MCF7 cells were obtained from ATCC and grown in high-glucose Dulbecco's modified Eagle's medium (DMEM) containing 10\% fetal bovine serum (FBS). HCT116 p53 $3^{+/+}$and HCT116 p53 $^{-/-}$isogenic human colorectal cancer cells were kindly provided by Bert Vogelstein (Johns Hopkins University, Baltimore, MD) and were grown in McCoy's 5A media containing $10 \%$ FBS. Wild-type mouse embryonic fibroblasts (MEFs) were kindly provided by Jean Wang (University of California at San Diego, San Diego, CA). p53 ${ }^{-1-}$ and $\mathrm{p} 53^{-/} \mathrm{E} 2 \mathrm{~F} 1^{-/-} \mathrm{MEFs}$ were a kind gift of Dr Olenski Petrenko, SUNY Stony Brook, NY. MEFs were grown in high-glucose DMEM containing $10 \%$ FBS. All cell types were grown in a $37^{\circ} \mathrm{C}$ humidified incubator containing $5 \% \mathrm{CO}_{2}$ and maintained at or below $80-90 \%$ confluence.

RNAi transfection/drug treatment. Cells were plated at the appropriate density to reach $40-50 \%$ confluence in $24 \mathrm{~h}$ and transfections were performed using the X-tremeGENE reagent (Roche) according to the protocols supplied by the manufacturer. The RNA oligonucleotides used in MCF7 and HCT116 cells were generated by Dharmacon based on the human mRNA for PNUTS: PNUTS RNAi: CAGCUAAACU GGUGAAGCA or non-targeting RNAi: siCONTROL nontargeting siRNA no. 1 (Dharmacon) which has at least 4 mismatches with all known human, mouse or rat genes which is sufficient to eliminate non-specific silencing of genes with similar sequences. Mouse specific interfering RNA was obtained from Santa Cruz Biotechnology for the homologous mouse mRNA PNUTS sequence (siRNA-SC61378) which was used in MEF experiments. Transfection mixtures (containing final concentration $100 \mathrm{nM}$ of RNA) were added to the cells. Twenty-four hours later cells were counted or underwent apoptosis assays or were lysed for protein analysis. For drug treated cells, Roscovitine (Calbiochem) was diluted in DMSO and cells were exposed to $25 \mu \mathrm{M}$ Roscovitine for 8 h. Control cells received DMSO alone.

Cell proliferation/apoptosis assays. Cell viability was determined using the Quick Cell proliferation assay kit (Biovision) based on the cleavage of the tetrazolium salt WST-1 to formazan. Percentage of viability was determined by comparing the number of viable cells in treated cultures to the number of viable cells in controls after subtraction of background values from each. The Cell Death Detection ELISA (Roche) was performed as directed by the manufacturer. Briefly, $10^{4}$ cells from each condition were lysed and subjected to a slow spin centrifugation to pellet nuclei. Extracts from the cytoplasmic fraction were used to detect fragmented DNA. For TUNEL analysis cells were plated on chamber slides at a density of 5,000 cells $/ \mu 1$. Cells were fixed in $4 \%$ para-formaldehyde for $1 \mathrm{~h}$ at room temperature and the TUNEL assay was carried out as per manufacturer's instructions (Chemicon, ApopTag ${ }^{\circledR}$ Plus Fluorescein In Situ Apoptosis Detection kit). Results were analyzed by fluorescence microscopy using an Olympus BX60 fluorescence microscope equipped with a monochrome digital acquisition camera. Counts were obtained by counting on random fields and by scoring at least 500 cells per experimental group.

Immunoblotting. SDS-PAGE and Western blotting was performed as previously described (13). In this study we utilized the following primary antibodies: PNUTS (Transduction Laboratories); Rb-phospho-780, Rb-phospho-795, Rb-phospho807/811, cleaved PARP, BAD, MCL-1, BAK, BID, Bcl-xL, Bcl-2 (Cell Signaling Technology); Rb (G3-245), unphosphorylated Rb (608) (Pharmingen), Rb-phospho-821 (Biosource), and B-actin (Sigma).

\section{Results}

PNUTS knockdown enhances Roscovitine-induced reduction in cell number. It has been previously shown that PNUTS knockdown or Roscovitine alone can cause a reduction in cell number $(19,26)$. To determine whether combined depletion of cdk activity by Roscovitine and activation of PP1 activity by PNUTS siRNA would exert a combinatorial effect on the cells, we treated $\mathrm{p} 53^{+/+}$and $\mathrm{p} 53^{-/}$HCT116 colon cancer cells for $8 \mathrm{~h}$ with Roscovitine $(25 \mu \mathrm{M})$ followed by knockdown of PNUTS as described in the Materials and methods. Viable cell number was measured $24 \mathrm{~h}$ following transfection of PNUTS siRNA. We observed that knockdown of PNUTS alone or Roscovitine treatment alone reduced cell number by approximately $30 \%$ in $\mathrm{p} 53^{+/+}$cells, with less of an effect in p53 ${ }^{-/}$cells (Fig. 1A). However, when cells were subjected to both treatments, cell number in $\mathrm{p} 53^{+/+}$cells was reduced by $80 \%$ and cell number in p53/- cells was reduced by $50 \%$. The efficacy of silencing PNUTS gene expression in these experiments is shown (Fig. 1B). 
A

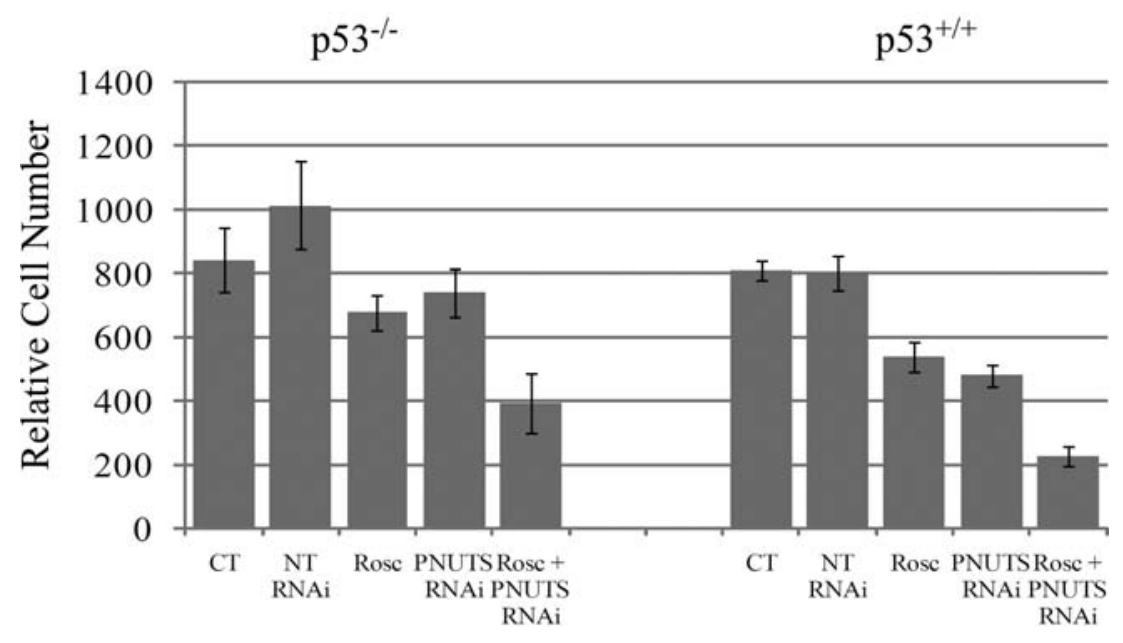

B

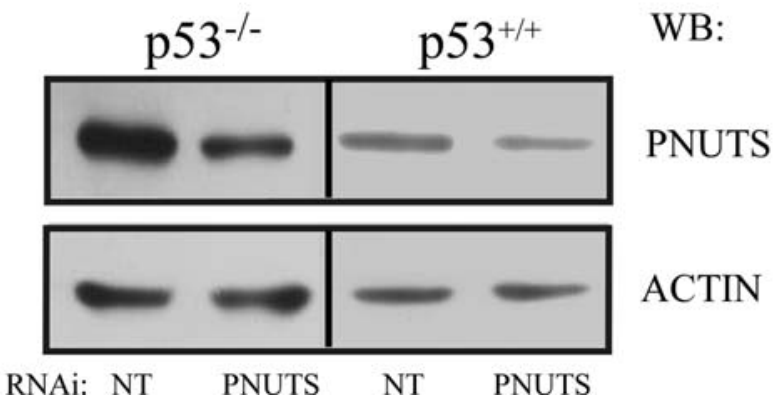

Figure 1. PNUTS knockdown increases the effect of Roscovitine on cell number in human cancer cells. (A) For combined treatments of Roscovitine (Rosc)

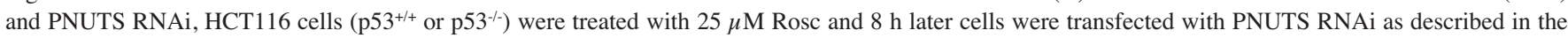
Materials and methods. Twenty-four hours after transfection cells were counted. For Rosc treatment alone, $8 \mathrm{~h}$ after addition of drug, media was replaced and cells were incubated for $24 \mathrm{~h}$ before counting was performed. For PNUTS RNAi alone, cells were treated with control non-targeting RNAi (NT) or PNUTS RNAi $24 \mathrm{~h}$ before cell counts were performed. Error bars represent standard deviation of the mean of triplicate samples. Data shown is representative of three independent experiments. (B) Cell lysates obtained from cell counting experiments were subjected to immunoblotting. RNAi using a non-targeting control sequence (NT) or PNUTS RNAi was utilized. PNUTS knockdown and equal loading were confirmed in p53\% and p53 $3^{+/+}$HCT116 cells by examining expression of PNUTS and B-actin, respectively. The antibodies utilized are indicated to the right of the figure.

PNUTS knockdown enhances cell death induced by Roscovitine. Previous studies utilizing siRNA to the PP1 targeting subunit PNUTS showed that dephosphorylation of Rb led to apoptosis in breast and colon cancer cells (19). Also, it has been shown that Roscovitine can cause cell death $(28,29)$. Therefore, in these experiments we measured whether the reduction in cell number observed when PNUTS siRNA was combined with Roscovitine led to an increase in cell death. We utilized MCF7 breast cancer cells which are $\mathrm{p} 53^{+/+}$and measured apoptosis by TUNEL assay. As shown in Fig. 2A, the apoptosis measured in controls (DMSO or NT-nontargeting RNAi) was normalized to 1 . Roscovitine and PNUTS RNAi separately increased the induction of apoptosis by 2 -fold, which was similar to results published previously (19). However, when MCF7 cells were treated first with Roscovitine $(25 \mu \mathrm{M}, 8 \mathrm{~h})$ followed by PNUTS siRNA $(24 \mathrm{~h})$ there was a 4-fold induction of apoptosis. The knockdown of PNUTS in MCF7 cells is shown (Fig. 2B). We next utilized p53 $3^{--}$HCT116 colon cancer cells and observed similar results using a cell death ELISA (Roche) to measure apoptosis (Fig. 2C). Thus the reduction in cell number observed when cells are treated with both Roscovitine and PNUTS siRNA is due to activation of apoptosis.

Apoptosis induced by PNUTS knockdown is dependent on E2F1. We next attempted to compare the mechanisms involved in apoptosis resulting from Roscovitine and/or PNUTS knockdown. Because Roscovitine and PNUTS siRNA each alter the phosphorylation state of $\mathrm{Rb}(19,25)$, and Rb phosphorylation regulates the association of $\mathrm{Rb}$ with $\mathrm{E} 2 \mathrm{~F} 1(37,38)$, we investigated the role of E2F1 in this series of experiments. Because it has clearly been shown that E2F1 is required for Roscovitine to induce apoptosis (36) we focused on the role of E2F1 in apoptosis induced by PNUTS knockdown. We utilized immortalized mouse embryonic fibroblasts that were either wild-type, $\mathrm{p} 53^{---}$, or DKO p53 $3^{--\mathrm{E}} 2 \mathrm{~F} 1^{-/}$. Knockdown of 


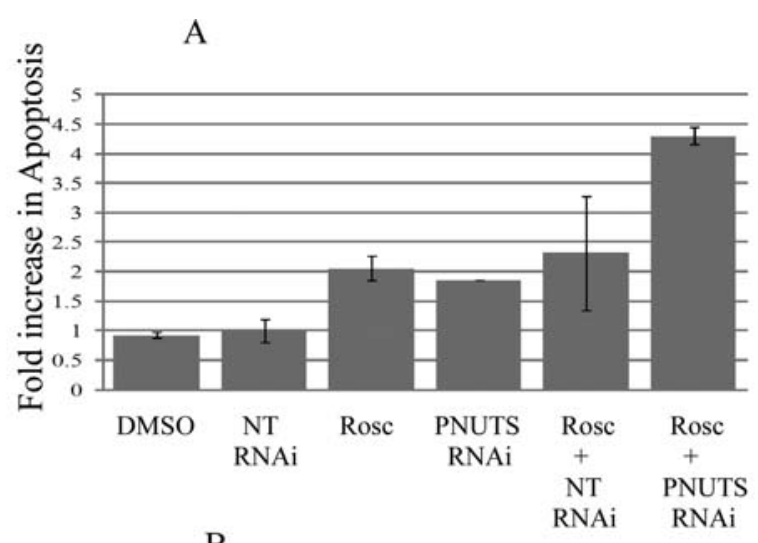

B
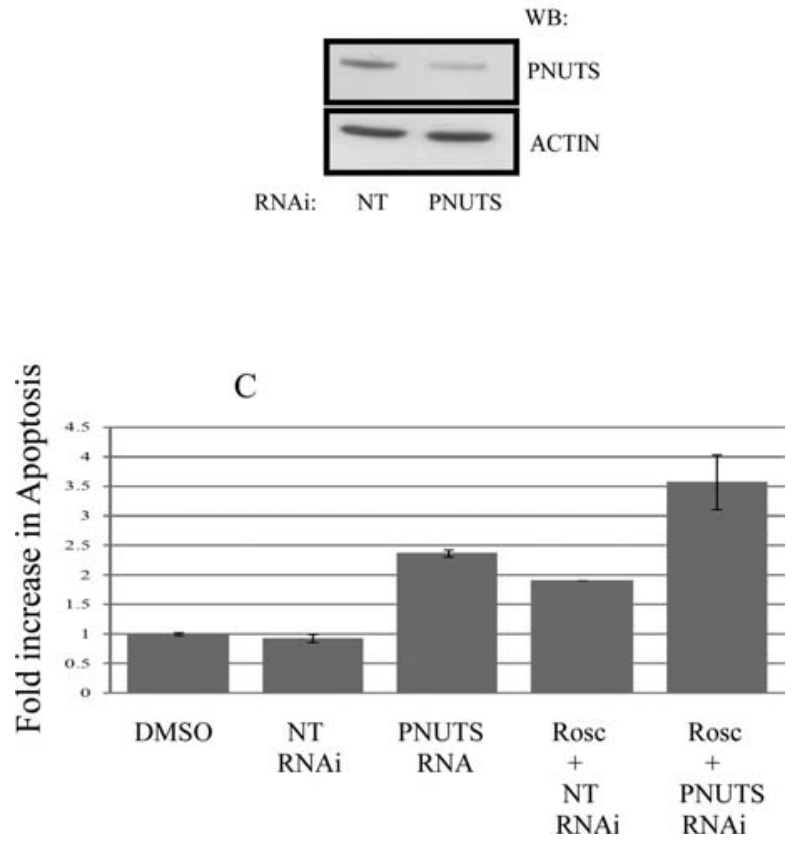

Figure 2. PNUTS knockdown increases the apoptotic effect of Roscovitine in cancer cells. (A) MCF7 cells were treated as control (DMSO or nontargeting RNA) or transfected with PNUTS RNAi or treated with Rosc or these treatments were combined as described in Fig. 1. Analysis was performed by TUNEL assay as described in the Materials and methods. The amount of apoptosis detected in control cells (DMSO) was normalized to one. The graph depicts the fold increase in degraded DNA observed in the cells under each condition. Error bars represent standard deviation of the mean of triplicate samples and data shown is representative of two independent experiments. (B) PNUTS knockdown and equal loading were confirmed in MCF7 cells by examining expression of PNUTS and ß-actin, respectively. The antibodies utilized are indicated to the right of the figure. (C) Following treatment of $\mathrm{p}^{-/-}$HCT116 cells as described in Fig. 1, apoptosis was measured by a Cell Death Detection ELISA which detects degraded DNA released from the nucleus into the cytoplasm. The amount of apoptosis (degraded DNA) detected in control DMSO treated cells was normalized to one. The graph depicts the fold increase in degraded DNA observed due to PNUTS RNAi. Error bars represent standard deviation of the mean of triplicate samples and data shown are representative of two independent experiments.

PNUTS was performed as described in the Materials and methods and reduced expression of PNUTS in these experiments is shown (Fig. 3). Whereas PNUTS siRNA caused a $30 \%$ increase in apoptosis in WT cells, and a $25 \%$ increase in p53 ${ }^{--}$MEFs, in $\mathrm{p} 53^{--}$E2F1/- DKO MEFs PNUTS knockdown caused only a $13 \%$ increase in apoptosis. Thus, the loss of

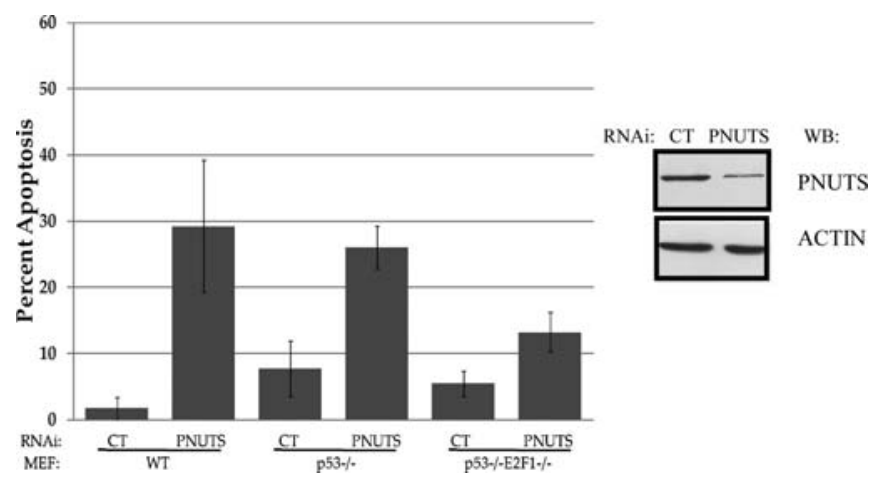

Figure 3. Apoptotic effect of PNUTS knockdown is dependent on E2F1. Wild-type (WT), $\mathrm{p} 53^{-/-}$and $\mathrm{p} 53^{-/} \mathrm{E} 2 \mathrm{~F} 1^{-/}$mouse embryonic fibroblasts (MEFS) were transfected with non-targeting (control-CT) or PNUTS RNAi and $24 \mathrm{~h}$ later apoptosis was measured by TUNEL assay as described in the Materials and methods. Error bars represent standard deviation of the mean of triplicate samples and data shown are representative of two independent experiments. Knockdown of PNUTS was verified by immunoblotting cells treated with either control (CT) or PNUTS RNAi.

E2F1 reduced the induction of apoptosis induced by PNUTS siRNA by $50 \%$. These data show that E2F1 is involved in the mechanism by which PNUTS knockdown leads to apoptosis. In addition, it supports our earlier study that showed that p53 is not involved in apoptosis induced by PNUTS knockdown (19).

$R b$ phosphorylation profiles in response to Roscovitine and PNUTS knockdown. To compare the effects of cdk inhibition vs. PP1 activation on Rb phosphorylation, we determined which sites of $\mathrm{Rb}$ were dephosphorylated in response to Roscovitine or PNUTS knockdown using p53/- HCT116 cells. Roscovitine inhibits kinase activity toward $\mathrm{Rb}$, while PNUTS siRNA activates phosphatase activity toward Rb. As shown in Fig. 4, these two strategies that cause Rb dephosphorylation display differences in specificity toward $\mathrm{Rb}$ phosphorylation sites. For example, Roscovitine treatment causes dephosphorylation of amino acid Ser-795 but not of Thr-821, Ser-780 or Ser-807/811 whereas PNUTS knockdown causes the dephosphorylation of Ser-795 as well as Thr-821, Ser-780, Ser-807/811, and Ser-608. Thus inhibition of cdk activity effects specific sites of Rb phosphorylation, and activation of PP1 toward Rb (via PNUTS knockdown) causes the dephosphorylation of a distinct set of amino acid sites.

Bcl-2 family protein expression profiles in response to Roscovitine and PNUTS knockdown. Because apoptosis is carried out by the bcl-2 family of proteins (39), we determined the effect of Roscovitine and PNUTS knockdown on the expression of several members of this family using p53 $3^{-/-}$HCT116 cells. Although neither Roscovitine or PNUTS knockdown effected the BAX or BID proteins as shown in Fig. 5, there were observable effects on other members of the group. Interestingly, Roscovitine dramatically reduced the expression of MCL-1, a finding which has previously been reported (28). However, PNUTS knockdown had no effect on the expression of this apoptosis mediator. Similarities in the effect produced by Roscovitine treatment and PNUTS knockdown include 


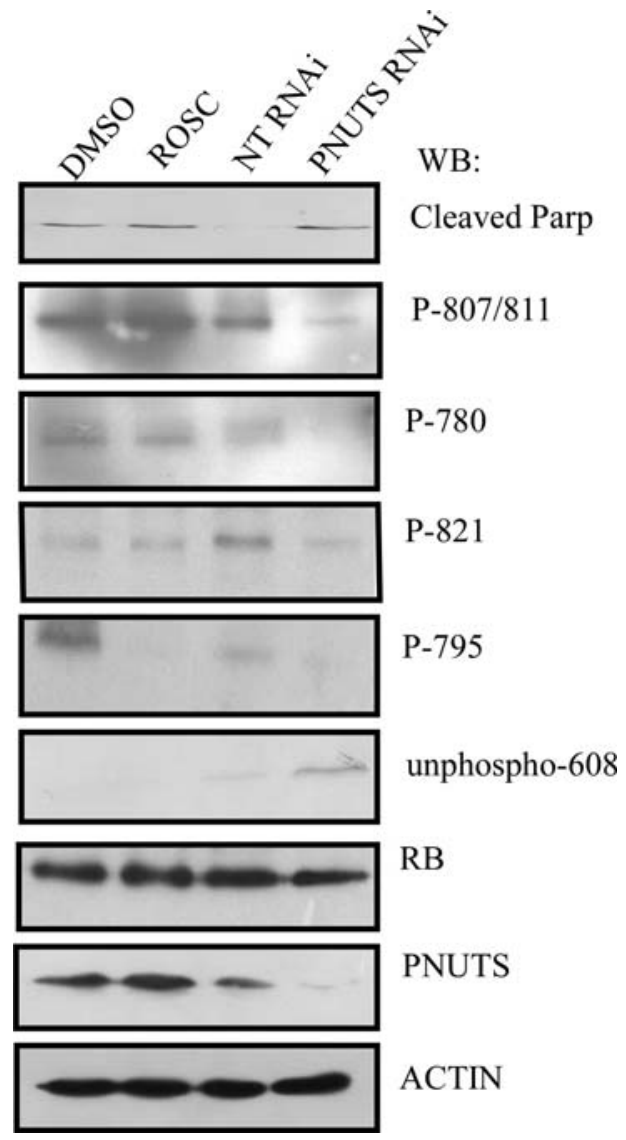

Figure 4. Effect of PNUTS knockdown vs. Roscovitine treatment on $\mathrm{Rb}$ phosphorylation state. p53 $3^{-/-}$HCT116 cells were treated with DMSO or Rosc for $8 \mathrm{~h}$ or with non-targeting (NT) or PNUTS RNAi for $24 \mathrm{~h}$ followed by immunoblotting as described in the Materials and methods. The antibodies utilized are indicated to the right of the figure. The antibodies to $\mathrm{Rb}$ amino acids $807 / 811,780,821$, and 795 recognize the phosphorylated sites of $R b$ However, the antibody to Rb-608 recognizes the unphosphorylated form of 608. Cleaved Parp is a marker of apoptosis (19). Total Rb expression is shown and PNUTS knockdown and equal loading were confirmed by examining expression of PNUTS and $B$-actin, respectively. Data shown are representative of two separate experiments.

the decrease of the anti-apoptotic protein Bcl-xL, and an increase in the pro-apoptotic proteins PUMA and BAD. Thus the effects of Roscovitine and PNUTS siRNA are similar on most of the bcl-2 family proteins with the notable exception of the effect on the expression of MCL-1.

\section{Discussion}

Alterations in the Rb pathway that lead to excessive phosphorylation of $\mathrm{Rb}$ have been observed in almost all cancer types $(3,40)$. These modifications include overexpression of cyclin proteins which activate cdks and/or the loss of endogenous cdk inhibitors (41). In this study we have examined the effect of targeting Rb phosphorylation in cancer cells. In previous studies we have shown that when $\mathrm{Rb}$ phosphatase is activated in cells using PNUTS siRNA, the $\mathrm{Rb}$ dephosphorylation that results leads to apoptosis (19). To further effect $\mathrm{Rb}$ phosphorylation, we utilized the cdk inhibitor Roscovitine. We found that utilized alone, Roscovitine or PNUTS siRNA caused a 2-fold increase in apoptosis in breast and colon cancer cells. However, when cells are exposed

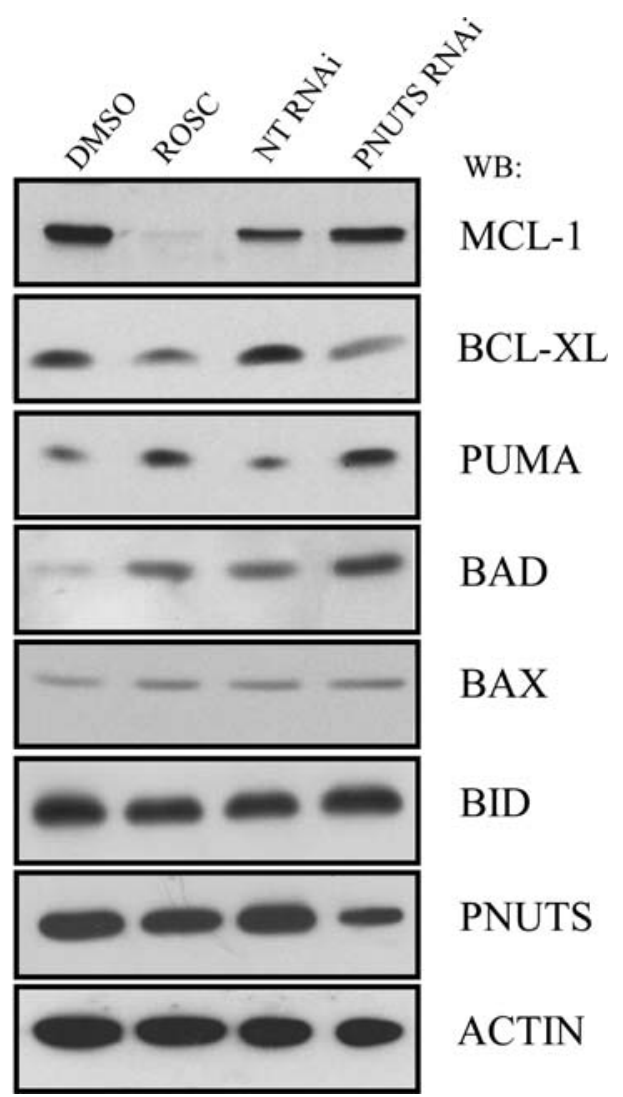

Figure 5. Effect of PNUTS knockdown vs. Roscovitine treatment on apoptosis regulators. p53 $3^{-/-}$HCT116 cells were treated with DMSO or Rosc for $8 \mathrm{~h}$ or with non-targeting (NT) or PNUTS RNAi for $24 \mathrm{~h}$ followed by immunoblotting as described in the Materials and methods. PNUTS knockdown and equal loading were confirmed by examining expression of PNUTS and B-actin, respectively. The antibodies utilized are indicated to the right of the figure. Data shown are representative of two separate experiments.

to both of these treatments, apoptosis is stimulated 4-fold. This increase in apoptosis induction observed when cells are treated with Roscovitine plus PNUTS siRNA suggests that the mechanism by which these two treatments act to induce apoptosis may be distinct.

Therefore, we analyzed the phosphorylation of $\mathrm{Rb}$ at several amino acids in order to determine whether activation of phosphatase activity (PNUTS siRNA) or inhibition of cdk activity (Roscovitine) led to similar or different phosphorylation profiles of $\mathrm{Rb}$. From this analysis of $\mathrm{Rb}$ phosphorylation, in these experiments, it appears that activation of phosphatase activity toward Rb (PNUTS knockdown) exerts a greater effect on $\mathrm{Rb}$ dephosphorylation than does cdk inhibition, evidenced by the increase in dephosphorylated sites on $\mathrm{Rb}$. This result suggests that in these experiments the primary effect of Roscovitine may not be the dephosphorylation of $\mathrm{Rb}$ but of other targets of the cdks as discussed below.

Because most of the tumor suppressor functions of $\mathrm{Rb}$ are thought to be mediated via the E2F family of transcription factors, we next determined whether E2F1 was involved in the apoptosis observed in these studies. E2F1 has been implicated in apoptosis that occurs through modulation of $\mathrm{Rb}$ activity (42). It is known that Roscovitine increases the 
expression of E2F1 (36). In addition, E2F1 siRNA reduces Roscovitine induced apoptosis in cells that lack p53 and in TRAIL induced apoptosis $(36,43)$. Finally inhibition of cdks by the cdk inhibitor flavopiridol causes dissociation of E2F from $\mathrm{Rb}$ and E2F1 activation (44). Thus it seems clear that Roscovitine does require the activity of E2F1 to cause apoptosis. In this study we determined that E2F1 is also required for apoptosis stimulated by activation of $\mathrm{Rb}$ phosphatase activity. This result further extends and strengthens our previous findings that $\mathrm{E} 2 \mathrm{~F} 1$ is involved in the mechanism by which PNUTS knockdown leads to apoptosis (19).

To understand the roles of the bcl-2 family of proteins in the mechanisms by which PNUTS knockdown and Roscovitine induce apoptosis, we evaluated the expression of this class of proteins after each treatment. The effects of both treatments on three apoptosis regulators were similar for both PNUTS knockdown and Roscovitine treatment. Bcl-xL is an inhibitor of apoptosis (39) and was diminished in expression by both treatments. PUMA is an activator of apoptosis (45) and its expression was increased by the two treatments. Finally the expression of pro-apoptotic BAD (39) was also elevated by both the treatments. However, a striking difference occurred in MCL-1 expression between cdk inhibition and phosphatase activation. A reduction in MCL-1 levels alone can induce apoptosis (28). Thus it was striking to observe that Roscovitine abolished the expression of MCL-1 whereas PNUTS knockdown had no effect on MCL-1 expression. It has been shown that the effects of Roscovitine on cells can be mediated through the inhibition of phosphorylation of cdk substrates such as RNA polymerase II. Reduced phosphorylation of RNA polymerase II leads to inhibition of transcription, which results in a loss of MCL-1 expression (28). Thus, in this study, it is likely that Roscovitine is acting via the inhibition of RNA polymerase activity, and not only through dephosphorylation of $\mathrm{Rb}$.

Although the involvement of p53 in the apoptosis observed in this study was not directly assessed, it is of note that loss of p53 in MEFs did not diminish the apoptosis observed when PNUTS expression was reduced (Fig. 3), suggesting that p53 is not required for PNUTS knockdown to cause apoptosis. This result is in agreement with our previous studies that showed that PNUTS knockdown was equally proficient in causing apoptosis in $\mathrm{p} 53^{+/+}$or $\mathrm{p} 53^{-/-} \mathrm{HCT} 116$ colon cancer cells (19). However, p53 likely plays a role in Roscovitine induced apoptosis. Recent studies have shown that Roscovitine up-regulates p53 and induces apoptosis (46). However, here we show that even in the absence of p53, Roscovitine clearly blocks the expression of MCL-1, suggesting that p53 may not be required for Roscovitine to cause cell death. Further studies should elucidate the role of p53 in Roscovitine-mediated cell death.

\section{Acknowledgements}

This study was supported by NIH CA120019 awarded to N. Krucher.

\section{References}

1. Taylor RC, Cullen SP and Martin SJ: Apoptosis: controlled demolition at the cellular level. Nat Rev Mol Cell Biol 9: 231-241, 2008 .
2. Hanahan D and Weinberg RA: Hallmarks of cancer. Cell 100: 57-70, 2000.

3. Mittnacht S: The retinoblastoma protein - from bench to bedside. Eur J Cell Biol 84: 97-107, 2005.

4. Clarke AR, Maandag ER, van Roon M, van der Lugt NM, van der Valk M, Hooper ML and Berns A: Requirement for a functional Rb-1 gene in murine development. Nature 359: 328-330, 1992.

5. Jacks T, Fazeli A, Schmitt EM, Bronson RT, Goodell MA and Weinberg RA: Effects of an Rb mutation in the mouse. Nature 359: 295-300, 1992.

6. Lee EYHP, Chang CY, Hu N, Wang YCJ, Lai CC, Herrup K, Lee $\mathrm{WH}$ and Bradley $\mathrm{A}$ : Mice deficient for $\mathrm{Rb}$ are nonviable and show defects in neurogenesis and haematopoiesis. Nature 359: 288-294, 1994.

7. Haas-Kogan D, Kogan SC, Levi D, Dazin P, T'Ang A, Fung YK and Israel MA: Inhibition of apoptosis by retinoblastoma gene product. EMBO J 14: 461-472, 1995.

8. Knudsen KE, Booth D, Naderi S, Sever-Chroneos Z, Fribourg AF, Hunton IC, Feramisco JR, Wang JY and Knudsen ES: RBdependent S-phase response to DNA damage. Mol Cell Biol 20: 7751-7763, 2000

9. Sherr CJ: Cancer cell cycles. Science 274: 1672-1677, 1996.

10. Nelson DA, Krucher NA and Ludlow JW: High molecular weight protein phosphatase type 1 dephosphorylates the retinoblastoma protein. J Biol Chem 272: 4528-4535, 1997.

11. Ianquinta PJ and Lees JA: Life and death decisions by the E2F transcription factors. Curr Opin Cell Biol 19: 649-657, 2007.

12. Dou QP, An B and Will PL: Induction of a retinoblastoma phosphatase activity by anticancer drugs accompanies p53independent G1 arrest and apoptosis. Proc Natl Acad Sci USA 92: 9019-9023, 1995.

13. Krucher NA, Rubin E, Tedesco VC, Roberts MH, Sherry TC and De Leon G: Dephosphorylation of Rb (Thr-821) in response to cell stress. Exp Cell Res 312: 2757-2763, 2006.

14. Popowski M, Ferguson HA, Sion AM, Koller E, Knudsen E and Van Den Berg CL: Stress and IGF-1 differentially control cell fate through mammalian target of rapamycin (mTOR) and retinoblastoma protein (pRB). J Biol Chem 283: 28265-28273, 2008.

15. Cohen PT: Protein phosphatase 1- targeted in many directions. J Cell Sci 115: 241-256, 2000.

16. Allen PB, Kwon YG, Nairn AC and Greengard P: Isolation and characterization of PNUTS, a putative protein phosphatase 1 nuclear targeting subunit. J Biol Chem 273: 4089-4095, 1998.

17. Kim YM, Watanabe T, Allen PB, Kim YM, Lee SJ, Greengard P, Nairn AC and Kwon YG: PNUTS, a protein phosphatase 1 (PP1) targeting subunit. Characterization of its PP1 and RNA binding domains and regulation by phosphorylation. J Biol Chem 278: 13819-13828, 2003.

18. Udho E, Tedesco V, Zygmunt A and Krucher NA: PNUTS (PP1 nuclear targeting subunit) inhibits retinoblastoma-directed PP1 activity. Biochem Biophys Res Commun 297: 463-468, 2002.

19. De Leon G, Sherry TC and Krucher NA: Reduced expression of PNUTS leads to activation of Rb-phosphatase and caspasemediated apoptosis. Cancer Biol Ther 7: 833-841, 2008.

20. Lee SJ, Lim CJ, Min JK, Lee JK, Kim YM, Won MH, Lee JY and Kwon YG: Protein phosphatase 1 nuclear targeting subunit is a hypoxia inducible gene: its role in post-translational modification of p53 and MDM2. Cell Death Diff 14: 1106-1116, 2007.

21. Malumbres M, Pevarello P, Barbacid M and Bischoff JR: CDK inhibitors in cancer therapy: what is next? Trends Pharmacol Sci 29: 16-21, 2008.

22. Meijer L, Borgne A, Mulner O, Chong JP, Blow JJ, Inagaki N, Inagaki M, Delcros JG and Moulinoux JP: Biochemical and cellular effects of roscovitine, a potent and selective inhibitor of the cyclin-dependent kinases cdc2, cdk2 and cdk5. Eur J Biochem 243: 527-536, 1997.

23. McClue SJ, Blake D, Clarke R, Cowan A, Cummings L, Fischer PM, Mackenzie M, Melville J, Stewart K, Wang S, Zhelev N, Zheleva D and Lane DP: In vitro and in vivo antitumor properties of the cyclin dependent kinase inhibitor CYC202 (roscovitine). Int J Cancer 102: 463-468, 2002.

24. Fischer PM and Gianella-Borradori A: CDK inhibitors in clinical development for the treatment of cancer. Expert Opin Investig Drugs 12: 955-970, 2003.

25. Whittaker SR, Walton MI, Garrett MD and Workman P: The cyclin-dependent kinase inhibitor CYC202 (R-Roscovitine) inhibits retinoblastoma protein phosphorylation, causes loss of cyclin D1, and activates the mitogen-activated protein kinase pathway. Cancer Res 64: 262-272, 2004. 
26. Whittaker SR, te Poele RH, Chan F, Linardopoulos S, Walton MI, Garrett MD and Workman P: The cyclin-dependent kinase inhibitor seliciclib (R-roscovitine; CYC202) decreases the expression of mitotic control genes and prevents entry into mitosis. Cell Cycle 6: 3114-3131, 2007.

27. Payton M, Chung G, Yakowec P, Wong A, Powers D, Xiong L, Zhang N, Leal J, Bush TL, Santora V, Askew B, Tasker A, Radinsky R, Kendall R and Coats S: Discovery and evaluation of dual cdk1 and cdk2 inhibitors. Cancer Res 66: 4299-4308, 2006.

28. MacCullem DE, Melville J, Frame S, Watt K, Anderson S and Gianella-Borradori A: Seliciclib (CYC202, R-Roscovitine) induces cell death in multiple myeloma cells by inhibition of RNA polymerase II-dependent transcription and down regulation of mcl-1. Cancer Res 65: 5399-5407, 2005.

29. Wesierska-Gadek J, Borza A, Walzi E, Krystof V, Maurer M, Komina O and Wandl S: Outcome of treatment of human HeLa cervical cancer cells with Roscovitine strongly depends on the dosage and cell cycle status prior to treatment. J Cell Biochem 106: 937-955, 2009

30. Dey A, Wong ET, Cheok CF, Tergainkar V and Lane DP: RRoscovitine simultaneously targets both the p53 and NF- $\mathrm{KB}$ pathways and causes potentiation of apoptosis:implications in cancer therapy. Cell Death Diff 15: 263-273, 2008.

31. Paprskarova M, Krystof V, Jorda R, Dzubak P, Hajduch M, Wesierska-Gadek J and Strnad M: Functional p53 in cells contributes to the anticancer effect of the cyclin-dependent kinase inhibitor roscovitine. J Cell Biochem 107: 428-437, 2009.

32. Lu W, Chen L, Peng Y and Chen J: Activation of p53 by roscovitine-mediated suppression of $\mathrm{mdm} 2$ expression. Oncogene 20: 3206-3216, 2001.

33. Fleming IN, Hogben M, Frame S, McClue SJ and Green SR: Synergistic inhibition of erbB signaling by combined treatment with seliciclib and ErbB-targeted agents. Clin Cancer Res 14: 4326-4335, 2008.

34. Lambert LA, Qiao N, Hunt KK, Lambert DH, Mills GB Meijer L and Keyomarsi K: Autophagy: a novel mechanism of synergistic cytotoxicity between doxorubicin and Roscovitine in a sarcoma model. Cancer Res 68: 7966-7974, 2008.
35. Wesierska-Gadek J, Maurer M and Schmid G: Inhibition of farnesyl protein transferase sensitizes human MCF7 breast cancer cells to Roscovitine-mediated cell cycle arrest. J Cell Biochem 102: 736-747, 2007.

36. Ortiz-Ferron G, Yerbes R, Eramo A, Lopez-Perez AI, De Maria R and Lopez-Rivas A: Roscovitine sensitizes breast cancer cells to TRAIL-induced apoptosis through a pleiotropic mechanism, Cell Res 18: 664-676, 2008.

37. Knudsen ES and Wang JYJ: Dual mechanisms for the inhibition of E2F binding to RB by cyclin-dependent kinase mediated RB phosphorylation. Mol Cell Biol 17: 5771-5783, 1997.

38. Brown V, Phillips RA and Gallie BL: Cumulative effect of phosphorylation of $\mathrm{pRB}$ on regulation of E2F activity. Mol Cell Biol 19: 3246-3256, 1999.

39. Cory S and Adams JM: The BCL2 family: regulators of the cellular life or death switch. Nat Rev Cancer 2: 647-656, 2002.

40. Sherr CJ and McCormick F: The RB and p53 pathways in cancer. Cancer Cell 2: 103-112, 2002.

41. Johansson M and Persson JL: Cancer therapy: targeting cell cycle regulators anti-cancer agents in medicinal chemistry. Anticancer Agents Med Chem 8: 723-731, 2008.

42. Tsai KY, Hu Y, Macleod KF, Crowley D, Yamasaki L and Jacks T: Mutation of E2F1 suppresses apoptosis and inappropriate $\mathrm{S}$ phase entry and extends survival of Rb-deficient mouse embryos. Mol Cell 2: 293-304, 1998.

43. Ma Y, Freeman SN and Cress WD: E2F4 deficiency promotes drug-induced apoptosis. Cancer Biol Ther 3: 1262-1269, 2004.

44. Jiang J, Matranga CB, Cai D, Latham VM, Zhang X, Lowel AM, Martelli F and Shapiro GI: Flavopiridol-induced apoptosis during $\mathrm{S}$ phase requires E2F1 and inhibition of cyclin A dependent kinase activity. Cancer Res 63: 7410-7422, 2003.

45. Yu J, Zhang L, Hwang PM, Kinzler KW and Vogelstein B: PUMA induces the rapid apoptosis of colorectal cells. Mol Cell 7: 673-682, 2001

46. Wesierska-Gadek J, Wandl S, Kramer MP, Pickem C, Krystof V and Hajek SB: Roscovitine upregulates p53 protein and induces apoptosis in human HeLaS (3) cervix carcinoma cells. J Cell Biochem 105: 1161-1171, 2008. 